\title{
A Comparison Study of Confinement Effect of PEDOT and CNT for Sulfur Nanocomposites in Li-S Battery Application
}

Nicholas Greco $^{1,2}$, Michael Ross ${ }^{1}$, Marc Hermann ${ }^{1}$, Yong Hao ${ }^{1 *}$

${ }^{1}$ Materials Engineering Department, California Polytechnic State University, 1 Grand Avenue, San Luis Obispo, CA 93407, United States. ${ }^{2}$ Mechanical Engineering Department, California Polytechnic State University, 1 Grand Avenue, San Luis Obispo, CA 93407, United States.

\author{
Article Details \\ Article Type: Research Article \\ Received date: $02^{\text {nd }}$ December, 2019 \\ Accepted date: $16^{\text {th }}$ December, 2019 \\ Published date: $18^{\text {th }}$ December, 2019
}

*Corresponding Author: Yong Hao, Materials Engineering Department, California Polytechnic State University, 1 Grand Avenue, San Luis Obispo, CA 93407, United States. E-mail: yhao@,calpoly.edu

Citation: Greco N, Ross M, Hermann M, Hao Y (2019) A Comparison Study of Confinement Effect of PEDOT and CNT for Sulfur nanocomposites in Li-S Battery Application. Cur Res Mater Chem 1: 105. doi: https://doi.org/10.33790/crmc1100105. Copyright: (C2019, This is an open-access article distributed under the terms of the Creative Commons Attribution License 4.0, which permits unrestricted use, distribution, and reproduction in any medium, provided the original author and source are credited.

\begin{abstract}
Lithium sulfur (Li-S) battery, a technology that has been studied since the 1980s, has yet to be brought into the commercial market. Current challenges to their implementation are namely: large volumetric expansion and shrinkage of $\mathrm{S}$ as the cell cycles, the diffusion and dissolution of polysulfides during cycling and sulfur inherently as an insulator. In an attempt to tackle the issues of sulfur as cathode, in this study, sulfur was synthesized via a chemical reaction altering elemental sulfur into nano-sized particles. Conductive polymer PEDOT was chosen for its flexible structure to contain the volumetric expansion of the sulfur $\neg$ nanoparticles (S-NPs). Multi-walled carbon nanotubes (MWCNTs) were selected as the matrix to localize the S-NPs. Polyvinyl-pyrrolidone (PVP), an amphiphilic compound, was used to optimize the size of S-NPs, containing them in a shell to ensure the S-NPs did not aggregate. Results show the system of S-NPs coated in PEDOT to be a suitable sulfur encapsulant, with a minimum $25 \mathrm{wt} \%$ of active material comprising the final structure.
\end{abstract}

\section{Introduction}

Traditional lithium-ion batteries are not viable for high-powered, portable electronics due to their low specific capacity, making them impossible, by either the economics or material limits, to integrate into efforts such as electric vehicles and large-scale storage operations $[1,2]$. The lithium-sulfur battery, with an energy density of 2,600 $\mathrm{Wh} / \mathrm{kg}$, a theoretical capacity of $1,675 \mathrm{mAh} / \mathrm{g}$ (both far greater than lithium-ion batteries), as well as a far less dangerous and expensive method of procurement and transport an attractive alternative [3]. Three central drawbacks to a sulfur cathode, however, are significant enough to define the majority of current Li-S battery research. Central to the issue of a functional battery is sulfur's non-conductive nature, leading to poor active material utilization. While charging and discharging, the active material utilized by the battery undergoes volumetric changes, facilitate lithium polysulfide $\left(\mathrm{LiS}_{\mathrm{n}}\right)$ diffusion, causing anode degradation and poor life cycling [4]. Finally, owing to the so called "shuttle effect", the diffusion of polysulfide material that gradually coats the anode of the Li-S sulfur battery to the point of ineffective operation requires that sulfur be encapsulated to prevent such degradation [4].

The methods of stabilizing S cathode structure were investigated by previous researchers. Mitigating sulfur's polysulfide diffusion, and thus retaining high-order polysulfides, was managed by W. Li et al. through a process centered around a surfactant intended to house the hydrophobic sulfur in nano-sized spheres [5]. Using a "single step, bottom-up approach", Li and his counterparts coat sulfur in a shell of polyvinylpyrrolidone (PVP) by exposing a solution of sodium thiosulfate and PVP to hydrochloric acid $(\mathrm{HCl})$. This method utilizes the auto-organization tendencies of PVP to form hollow microspheres, which are then infiltrated by sulfur, which precipitates into the sphere during the reaction of sodium thiosulfate and $\mathrm{HCl}$. The attraction of the hydrophobic sulfur to the hydrophobic tails of the PVP instigates the precipitation of sulfur. The results show uniform, semi-hollow spheres with the average size of $400-500 \mathrm{~nm}$ and empty space allowing sulfur to expand inward instead of outward. It reveals nanosized sulfur can overcome the barriers to ion and electron transport which is proven in the reported high specific discharge capacities of $1,179 \mathrm{mAh} / \mathrm{g}$ at $\mathrm{C} / 10$. Li et al. investigated the feasibility of a carbon nanotube foam lattice to serve as the conductive medium to overcome sulfur's insulating tendencies [1]. The low density, preformed carbon lattice is synthesized from water dispersed CNTs. Once the foam is created, it is infiltrated with sulfur, vaporized at $175^{\circ} \mathrm{C}$, causing the sulfur to nucleate and grow on and inside the CNT foam, a direct result of capillary effect. This method produces uniform loading of the sulfur into the structure at a more rapid pace than liquid-based infiltration methods. Upon compression of the sulfur-infiltrated CNT foam, a dense structure is produced, exhibiting active material loading of $79 \mathrm{wt} \%$ and gravimetric capacity of $1039 \mathrm{mAh} / \mathrm{g}$. This approach differed from the traditional methods of melt infiltration, which disbands CNT-CNT pathways that prove very useful in amplifying the conductivity of the overall structure. $\mathrm{Li}$ et al. further extended their previous research on monodisperse Sulfur/PVP spheres, compare the feasibility of three separate conductive polymers in an effort to elucidate the electrochemical impact each polymer has during the cycling of a Li-S cathode [6]. The conductive polymers offer an alternative that may not only retain the polysulfides but also improve rate capabilities of the battery. In this study, polyaniline (PANI), polypyrrole (PPY) and Poly(3,4-ethylenedioxythiophene) (PEDOT) are coated onto the uniform Sulfur/PVP spheres mentioned previously before electrochemical testing was undertaken. Li et al. also examined the effect of coating thickness in both containing polysulfides and increasing active material loading. Their findings show that the cycle life and rate performance of PEDOT trumped those of PPY and PANI; after 500 cycles at $\mathrm{C} / 2$, the PEDOT polymer delivered a gravimetric capacity of $780 \mathrm{mAh} / \mathrm{g}$, with PPY exhibiting a gravimetric capacity of $726 \mathrm{mAh} / \mathrm{g}$ (PANI is not mentioned at this level of cycling). 
In this study, a three-pronged effort at synthesizing a sulfur cathode was made, aimed at addressing what research suggests to be the three major issues with the realization of commercializable Li-S battery technology. It is the understanding of the authors that no one of these issues can be addressed independent of the other issues without adverse impact on battery performance; our efforts reflect this supposition. In this work, elemental sulfur was chemically processed into nanoparticles coated in a surfactant PVP, which was then sonicated with polymerized EDOT to contain the sulfur during volumetric expansion and contraction without rupturing, while maintaining the electrical conductivity of CNT and PEDOT by different routes with different structures. The thickness of PEDOT wall is a key parameter in our investigation, as too much PEDOT reduces the cathode's specific capacity, while too little might be insufficient to contain the volumetric expansion of the S-NP. CNTs were sonicated into this mixture to serve as a conductive matrix. As a boon of the usage of a surfactant (PVP) in tandem with the conductive polymer PEDOT, structures 1 and 2 which employ these methods exhibit active material loading of approximately $25 \mathrm{wt} \%$ and display morphologies that indicated the effective encapsulation and nano-sizing of the elemental sulfur. Structure 3 exhibits the microstructures with agglomeration of sulfur particles in micron size which indicates PVP plays a big impact on the sulfur particle formation.

\section{Experimental}

\section{Synthesis of three types of sulfur composites}

The three types of structures are synthesized via three different routes as indicated in Figure 1. The S-NPs were fabricated via a chemical reaction decomposition method by using $5.0668 \mathrm{~g}$ sodium thiosulfate and $12 \mathrm{M}$ hydrochloric acid $(\mathrm{HCl}$ ) (see below for calculations). PVP of molecular weight $40000 \mathrm{M}$.W was dispersed in aqueous solution in the presence of the reacting $\mathrm{Na}_{2} \mathrm{~S}_{2} \mathrm{O}_{3}$ and $\mathrm{HCl}$ to allow for the chemically produced sulfur to infiltrate the PVP spheres [5]. The sample was magnetically stirred for 24 hours before being diluted with a $0.4 \mathrm{M}$ solution of PVP. EDOT was oxidatively polymerized in the presence of the active agents camphorsulfonic acid and ammonium persulfate [5]. The processing of each structure was done with both pulse and continuous sonication methods, with the continuous sonication method delivering substantially more watts to the solution [7]. The effect of this parameter will be discussed in the conclusions.

\section{Structure 1}

The multi-walled Carbon Nanotubes (MWCNTs, Outer Diameter: 20-30 nm, Length: 10-30 $\mu \mathrm{m}$ ) were purchased from Cheaptubes Inc. The structure 1 in Figure 1 is comprised of an S-NP solution created from the chemical reaction of sodium thiosulfate and $\mathrm{HCl}$, sonicated onto a lattice of MWCNTs, which is in turn sonicated with polymerized EDOT to produce the resulting structure. The process is initiated by the sonicating of $5.0595 \mathrm{~g}$ of sodium thiosulfate $\left(\mathrm{Na}_{2} \mathrm{~S}_{2} \mathrm{O}_{3}\right)$ with $12 \mathrm{M} \mathrm{HCl}$ in the presence of $2.23 \mathrm{~g}$ of PVP. The sonication takes place for 30 minutes at $80 \%$ amplitude. Following this, the PEDOT coating was introduced and a solution comprised of $38.1 \mathrm{mg}$ of MWCNTs dispersed in $50 \mathrm{~mL}$ of distilled water is sonicated with the S-NP@PEDOT structure for another 30 minutes at 80\% amplitude. This follows an overnight settling period and dilution with distilled water to a $\mathrm{pH} \sim 7$. The PEDOT coating was achieved by mixing 0.42 $\mathrm{g}$ of ammonium persulfate with $77 \mu \mathrm{L}$ of EDOT monomer in the presence of $0.0713 \mathrm{~g}$ of camphorsulfonic acid.

\section{Structures 2 and 3}

This structure reverses the order of structure 1, as shown in Figure 1, in that sulfur is sonicated onto a CNT lattice before the introduction of a PVP solution. The structure begins with the mixture of a $50 \mathrm{~mL}$ aqueous solution comprised of $38.1 \mathrm{mg}$ of CNTs plus $\sim 50 \mathrm{~mL}$ distilled water and $35 \mathrm{~mL}$ of $80 \mathrm{mM} \mathrm{Na}_{2} \mathrm{~S}_{2} \mathrm{O}_{3}$ in the presence of $12 \mathrm{M} \mathrm{HCl}$. The mixing occurs in the presence of continuous or pulse sonication as mentioned above. Following the introduction of the $\mathrm{Na}_{2} \mathrm{~S}_{2} \mathrm{O}_{3}$ solution with the CNTs and subsequent sonication for 30 min, $2.225 \mathrm{~g}$ of PVP is introduced into the solution, which is further sonicated for another $30 \mathrm{~min}$. Following an overnight settling period and dilution with distilled water to a $\mathrm{pH} \sim 7$, the PEDOT coating was introduced. This was achieved by mixing $0.4195 \mathrm{~g}$ of ammonium persulfate with $77 \mu \mathrm{L}$ of EDOT monomer in the presence of $0.0698 \mathrm{~g}$ of camphorsulfonic acid. The structure 3 of nanocomposite was synthesized as same as structure 2, but without PVP.

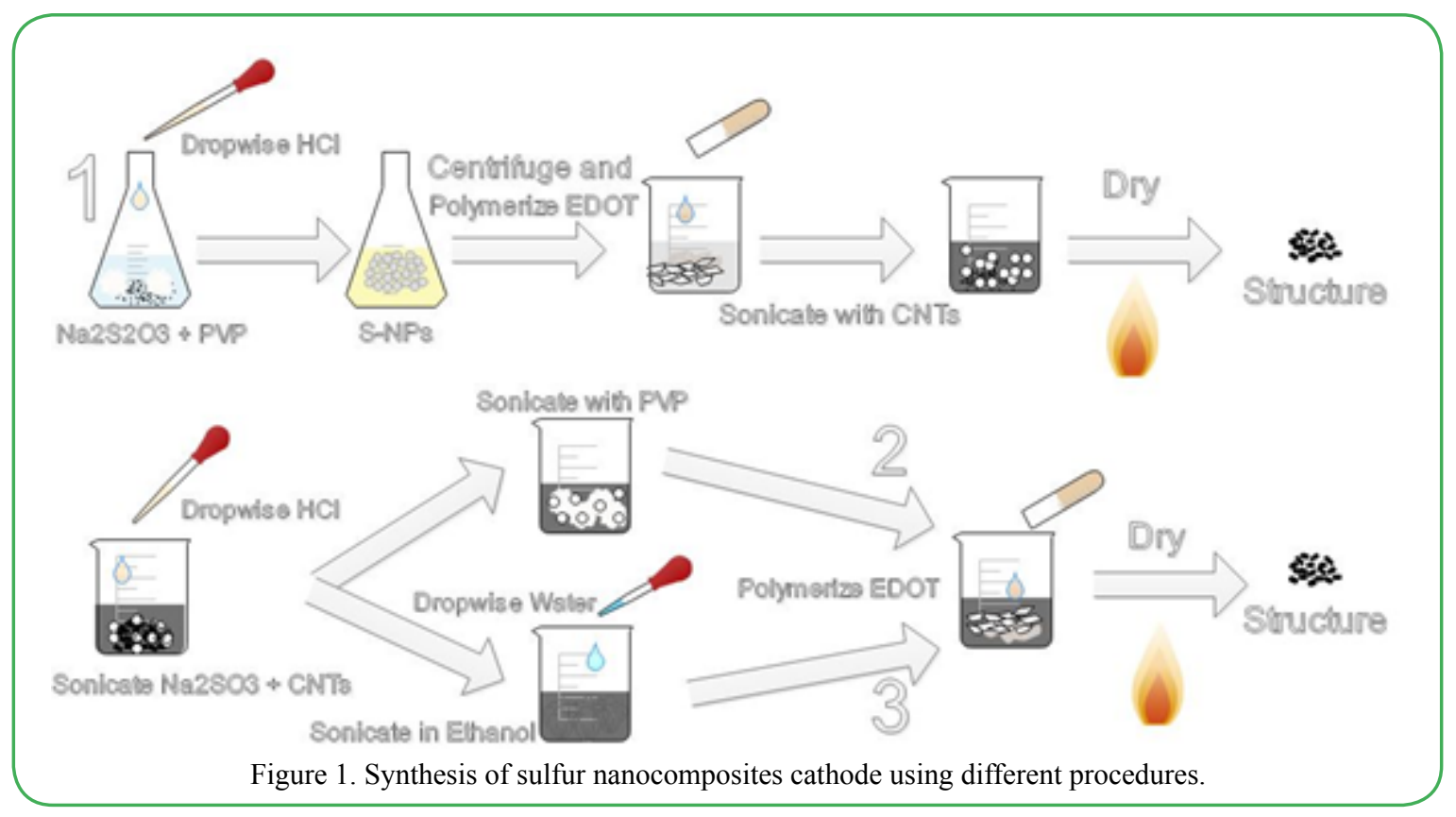




\section{Characterization measurements}

The morphology of the individual cathodes was examined using a FEI Quanta 2000 field-emission scanning electron microscopy (SEM). The composition of the cathodes was investigated by Thermogravimetric Analysis (TGA) using a Mettler Toledo TGA/ SDTA851. Further surface characterization of each nanocomposite was accomplished by Fourier transform infrared (FTIR) spectroscopy instrument using the JASCO FT/IR-4600.

\section{Results and Discussion}

Figure 2a describes a morphology of the S-NPs after being coated in PEDOT and sonicated with CNTs. The S-NPs are on the scale of between 0.5 and 3 microns in diameter and are relatively uniform in distribution. The size of these S-NP particles is owed to the introduction of PVP causing the formation of these spherical structures due to the hydrophobic nature of sulfur attracting the hydrophobic tails of the PVP. The formation of these spheres potentially includes hollow regions in which the sulfur can expand and thus improve the life cycle of a battery, as the containment of low order polysulfides would be achieved for longer. In figures $2 b$ and $2 c$, it can be observed that the spindly appearance of the CNTs. This is a direct result of the application of PEDOT prior to the introduction of the CNTs as the structural matrix and S/PEDOT spheres are embedded into the CNT matrix. A benefit of applying CNTs last is that the resulting structures have a higher surface area which would potentially benefit for the rate of ion transportation.

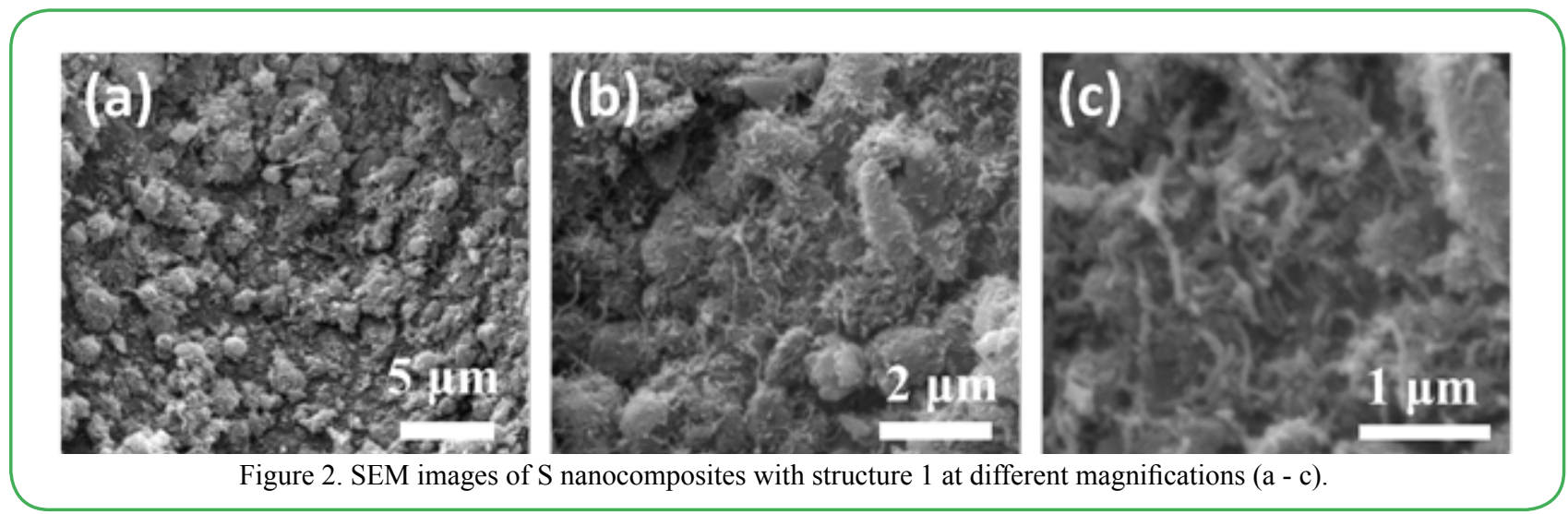

In Figure 3a, we observe a multitude of S-NP aggregated and mixed with PEDOT. The nanometer scale of the S-NPs indicates how the presence of PVP produces relatively uniform S-NP particles. The structure as a whole, despite the inclusion of CNTs, has an overall "smoothness", especially when compared to the jaggedness observable in Figure 2. This is attributed to the coating of PEDOT in the structure. In Figure $3 b$ and 3c, the encapsulated S-NPs are on the order of the nanometer/micron scale, ranging in size from 0.2 to 0.5 microns in span. Additionally, these particles display corallike structures, with void space in between adjacent S-NP particles. PEDOT appears to be enclosing the S-NP/CNT contents of the particle. These particles appear to undergo a cleavage of sorts, a result of the chemical processing steps to which the structure was subjected.

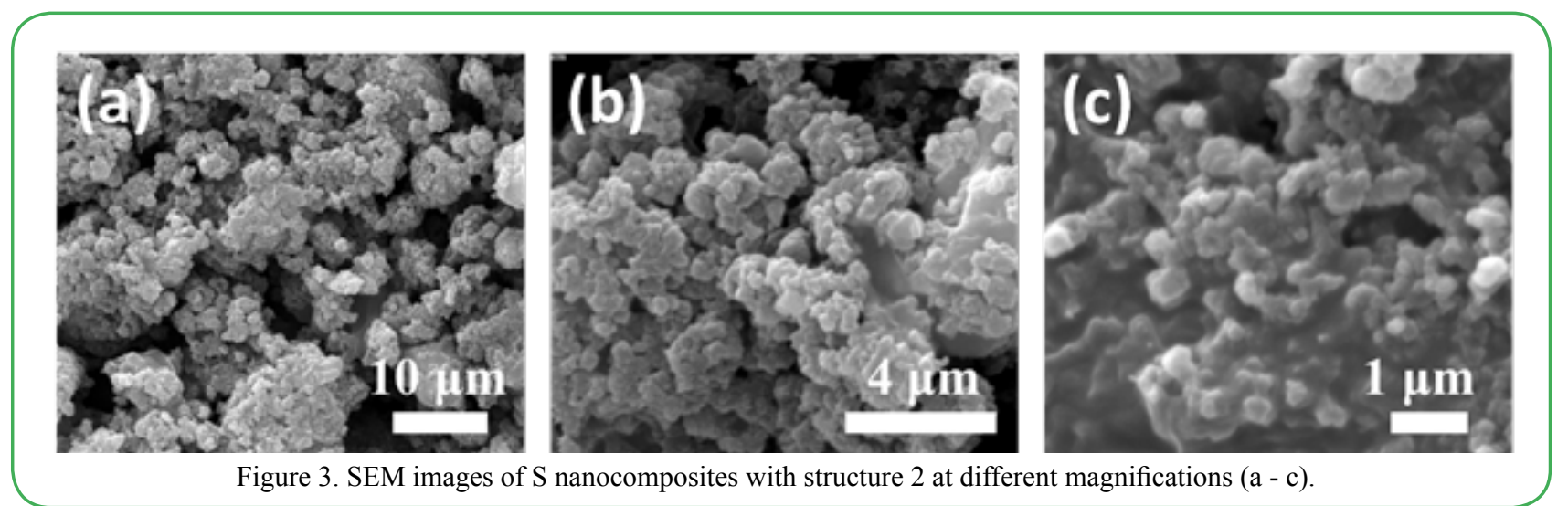

In Figure 4a, it can be observed that the average particle size is on a larger scale than the previous images. Additionally, the structure is similar to that of Figure 2a. The particles in this image lack uniformity in size and distribution. This can be attributed to the lack of PVP introduction after the processing of the elemental sulfur. Similarly, in figure $4 \mathrm{~b}$, the greater size of the particles is due to the lack of PVP included in the structure; micron size sulfur was sonicated with CNTs before being coated in PEDOT. Figure 4c shows the structure of the composite mixed with PEDOT. This emphasizes the importance of incorporating PVP when the elemental sulfur is initially processed; this structure is likely to have lowered the electrochemical performance as a result of the large agglomeration of S particles.

Figure 5 shows the TGA results of different samples. For structure 1 , no losses occur until around $200^{\circ} \mathrm{C}$, which indicates the proper drying of the structure before TGA analysis, as well as the strength

Cur Res Mater Chem

Volume 1.2019. 105 of the bonds between the hydrophobic region of the PVP and the elemental sulfur. Thus, when weight loss begins to occur at $200^{\circ} \mathrm{C}$, it is due to the evaporating of sulfur, hence the significant loss of material between 200 and $300^{\circ} \mathrm{C}$. The gradual loss in weight percent from approximately $325^{\circ} \mathrm{C}$ to $550^{\circ} \mathrm{C}$ is due to the evaporation of PVP/PEDOT. That said, the PEDOT that forms during this process evaporates at considerably higher temperatures. There is the sharp decrease that occurs at $\sim 560^{\circ} \mathrm{C}$. This decrease is contributed to losses from CNT oxidation [8]. For structure 2, initial losses at $\sim 40^{\circ} \mathrm{C}$ are likely due to the presence of residual ethanol used to dilute the solution. The large drop in mass beginning at $160^{\circ} \mathrm{C}$ is owed to the evaporation of sulfur [9]; the mass continues to drop at a similar rate until around $275^{\circ} \mathrm{C}$, as $P E D O T$ is evaporated from the composite as well. The final decrease from $275^{\circ} \mathrm{C}$ to $400^{\circ} \mathrm{C}$ is owed to the evaporation of PEDOT. Because the elemental sulfur coated in PVP was exposed to CNTs before PEDOT, the same PVP-PEDOT bonds 
that retained its composition until higher temperatures did not form, hence the loss of the majority of the weight of the structure at a lower temperature. For structure 3, the initial mass loss that starts at approximately $50^{\circ} \mathrm{C}$ and continues until approximately $200^{\circ} \mathrm{C}$ is owed to a combination of residuals, those being ethanol from washing the structure, distilled water for the same purpose, Subsequent mass loss from $\sim 210^{\circ} \mathrm{C}$ onward bears the evaporation of sulfur particles.
At this temperature, the evaporation of PEDOT is what as well will contribute to the majority of the mass loss striking resemblance to the PEDOT control curve; any deviation with the PEDOT control is due to its interface with the CNTs and possibly elemental sulfur. TGA results show $\mathrm{S}$ cathodes have minimum $25 \mathrm{wt} . \%$ active material of sulfur, with variable sulfur loading depending on polymers used.

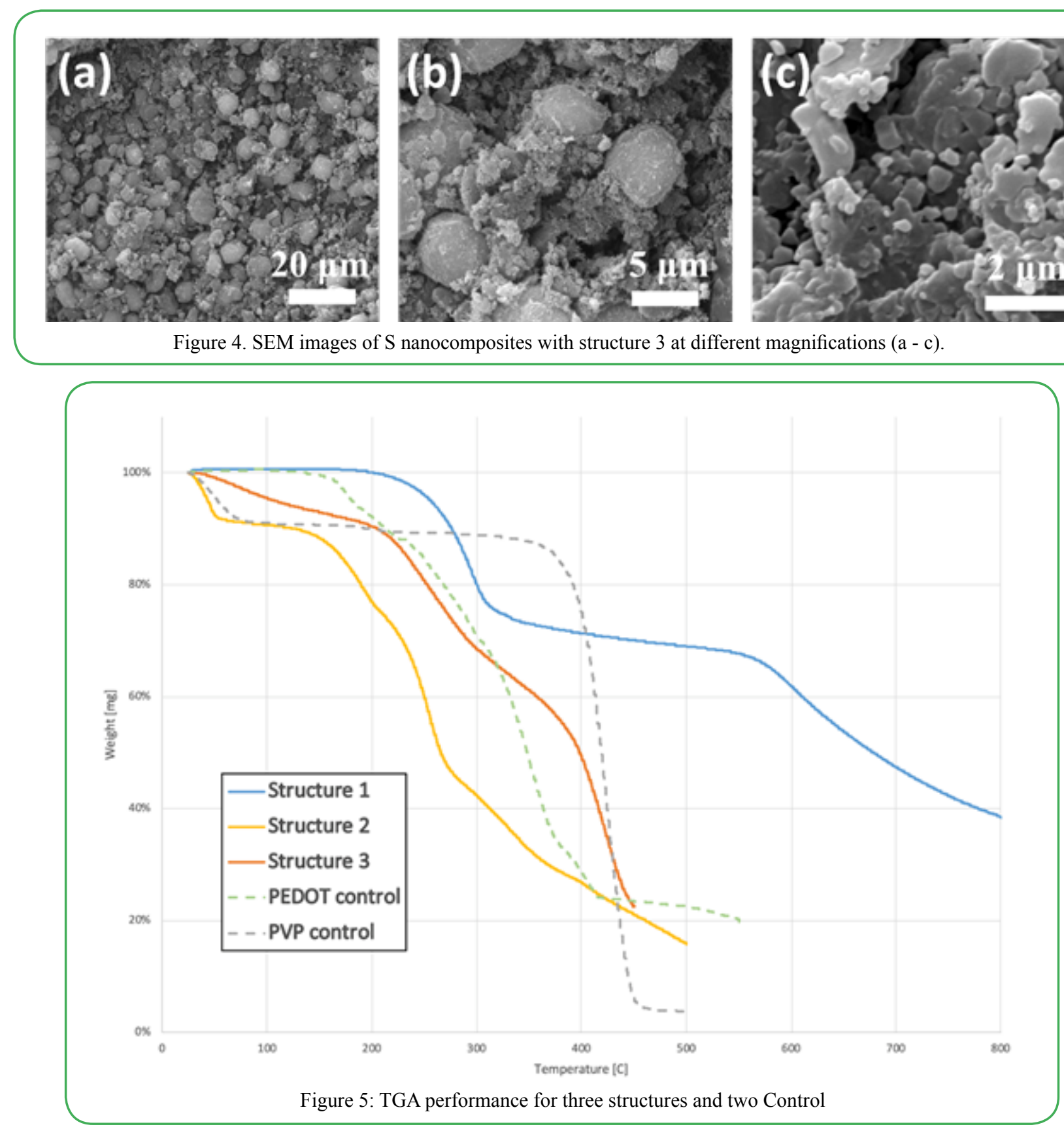

The FT-IR was tested for each structure and spectrums are shown in Figure 6. It can be observed that Structure 2 and Structure 3 share a number of the listed peaks above, which is attributable to the fact that they are both coated with PEDOT as a final step in the processing of the structure. As FT-IR is a spectroscopy tool useful for analyzing surfaces, it is logical that the results should resemble one another. Unfortunately, CNT loading of structure 1 prevented distinguishable peaks from being documented. The $668 \mathrm{~cm}^{-1}, 1289 \mathrm{~cm}^{-1}$, and 1559 $\mathrm{cm}^{-1}$ peaks observable in Structure 2 correspond with C-S, C-C, and $\mathrm{C}=\mathrm{C}$ bonds respectively. The peaks are likely a result of the thiophene ring in the EDOT monomer [10,11], indicating that PEDOT is in fact present in our structure. Additional evidence is observed in the $1079 \mathrm{~cm}^{-1}$ peak, a result consistent with C-O-C bond stretching in the ethylenedioxy group of EDOT [10]. While it is possible that the measured peaks could be the result of bonds formed by functionalized CNTs, the lack of peaks in Structure 1 and the strong correlation the observed peaks shown with published results on PEDOT make this unlikely. As such, it is reasonable to conclude that PEDOT successfully encapsulates the structure, an asset in the containment of lower order polysulfides [5]. Additionally, the 1418 $\mathrm{cm}^{-1}$ peak of structure 2 is characteristic of C-C bond stretching and the $611 \mathrm{~cm}^{-1}$ of structure 3 is indicative of sulfur bond stretching [11, 12]. The latter of these two peaks confirms the presence of sulfur within structure 3 , as there is PEDOT in structure 3 and thus no thiophene ring to undergo such stretching. 


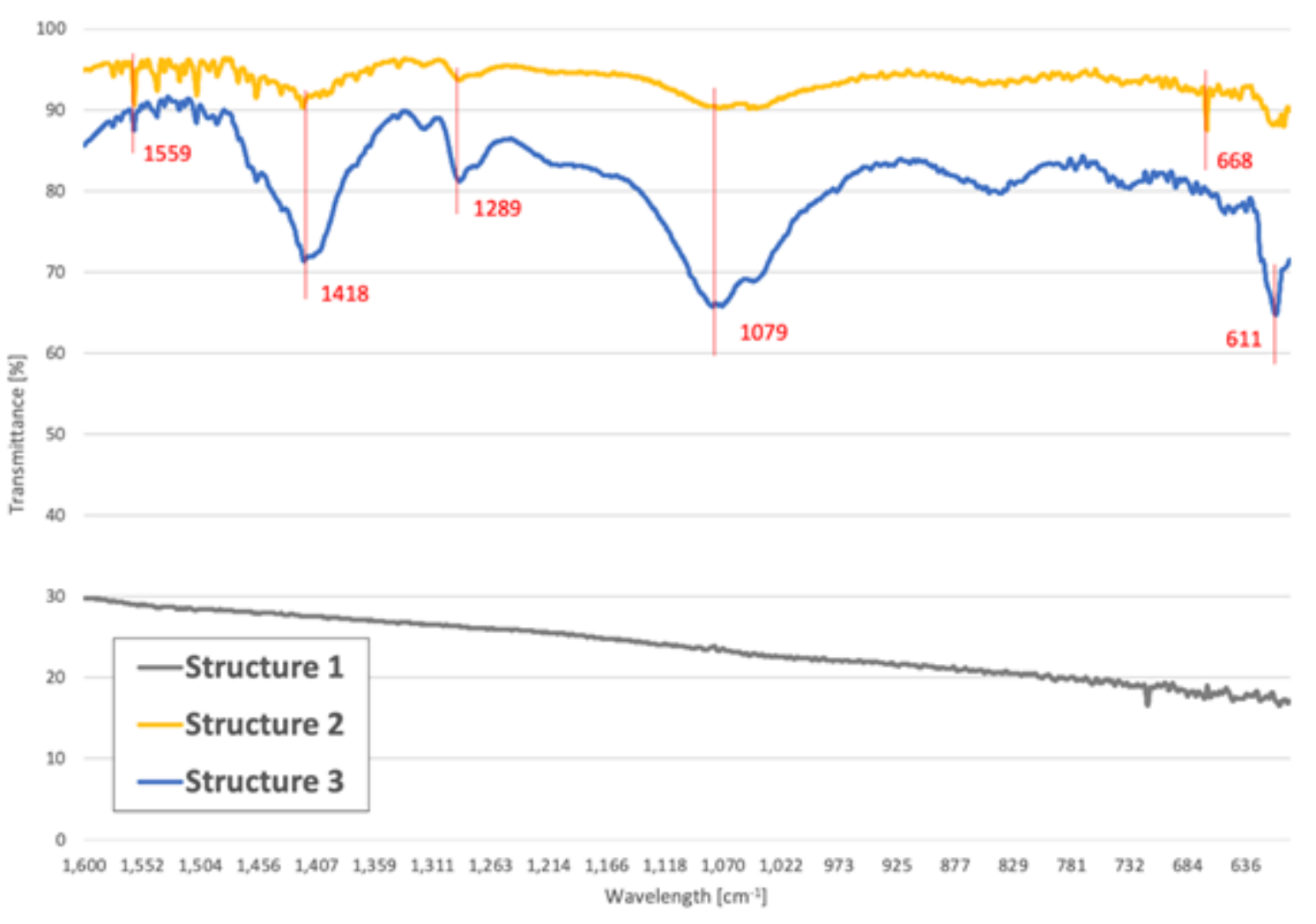

Figure 6: FT-IR spectrum for each cathode structure

\section{Conclusion}

In summary, three structures of varying in both composition and the order of their synthesis demonstrate the benefits of the dual employment of a surfactant and conductive polymer encapsulant. The presence of a surfactant, PVP in this work, produced uniform S-NPs, while PVP exclusion produced micron sized particles. Structure 1 is in a high surface area structure which would be beneficial as a Li-S cathode. The PEDOT coating of structure 2 would more specifically mitigate the lithium sulfide shuttle effect and is thus a predictably more effective encapsulant of the sulfur nanoparticles. The use of a surfactant such as PVP or Triton-X100 is recommended for nanoparticle fabrication. The fabrication of $\mathrm{S}$ composites with PEDOT as a final step will likely decrease interfacial resistance within the battery due to the conductive polymer coating. The active material loading of approximately $25 \mathrm{wt} \%$ could be optimized in future work to hold promise for a noteworthy gravimetric capacity. The synthesized $\mathrm{S}$ nanocomposites will be electrochemically evaluated in our future work.

\section{Acknowledgments}

The authors thank the Cal Poly Materials Engineering Department for use of their characterization equipment and facilities. The authors thank financial support of CENG Summer Undergraduate Research Program at Cal Poly.

Conflict of interest: The authors have declare no conflict of interest.

\section{References}

1. Li (2017) Sulfur Vapor-Infilitrated 3D Carbon Nanotube Foam for Binder-Free High Areal Capacity Lithium-Sulfur Battery Composite Cathodes, ACS Nano 4877-4884.

2. Lee J, Choi W (2015) Surface Modification of Sulfur Cathodes with PEDOT:PSS Conducting Polymer in Lithium-Sulfur Batteries, J The Electrochemical Soc 162: A935-A939.

3. Linda FN, Marine C, Quan P (2014) Lithium-sulfur batteries, MRS Bulletin 39: 436-442.

Cur Res Mater Chem

Volume 1.2019. 105
4. Manthiram A, Fu Y, Su Y (2012) Challenges and Prospects of Lithium-Sulfur Batteries, Accounts Chem Res 46: 1125-1134.

5. Li W, Zheng G, Yang Y, Seh Z, Liu N, et al. (2013) Highperformance hollow sulfur nanostructured battery cathode through a scalable, room temperature, one-step, bottom-up approach, Proceedings National Acad Sci 110: 7148-7153.

6. Weiyang L, Qianfan Z, Guangyuan Z, Zhi WS, Hongbin Y, et al. (2013) Understanding the Role of Different Conductive Polymers in Improving the Nanostructured Sulfur Cathode Performance, NanoLetters 5534-5540.

7. Sun L, Li M, Jiang Y, Kong W, Jiang K, et al. (2014) Sulfur Nanocrystals Confined in Carbon Nanotube Network As a Binder-Free Electrode for High-Performance Lithium Sulfur Batteries, Nano Letters 14: 4044-4049.

8. Culebras M, Gómez CM, Cantarero A (2013) J Mater Sci 48: 2855.

9. Dysart AD, Burgos JC, Mistry A, Chen CF, Liu Z, et al. (2016) Towards Next Generation Lithium-Sulfur Batteries: NonConventional Carbon Compartments/Sulfur Electrodes and Multi-Scale Analysis, J Electrochemical Soc 163: 730-741

10. Lee J, Choi W (2015) Surface Modification of Sulfur Cathodes with PEDOT:PSS Conducting Polymer in Lithium-Sulfur Batteries, J Electrochemical Soc.

11. Kiristi M, Bozduman F, Aysegul UO, Ahmed H, Lutfi o, et al. (2015) A Comparison Study of Microwave and Radio Frequency Plasma Polymerized PEDOT Thin Films, J Macromolecular Sci 124-129.

12. Ahmed (2019) Preparation of PVDF-TrFE based electrospun nanofibers decorated with PEDOT-CNT/rGO composites for piezo-electric pressure sensor, Journal of Materials Science: Materials in Electronics, Springer Nature. 